\title{
The Challenge of Blunt Abdominal Trauma in Children: Report of a Case and Review of Management
}

\author{
Ana Maria Minaya-Bravo a, b, Hector Aguado-Lopez ${ }^{\mathrm{a}}$
}

\begin{abstract}
Blunt abdominal trauma is the most frequent cause of abdominal injuries in children. The term solid organ injury (SOI) is referred to liver, spleen and kidney. Splenic injuries are the most common. The management of SOI has been motive of discussion for years and has undergone a radical shift in the last decades. Currently, non-operative management is the gold standard of care when circumstances are favorable. In 1977, non-operative management was supported by surgeons in Toronto. Later, the American Pediatric Surgical Association (APSA) pointed that management should be based on imaging findings and CT grading of the American Association for the Surgery of Trauma. However, this caused long stays and unnecessary follow-up imaging. Soon, APSA guidelines were questioned and raised controversy. Finally, hemodynamic status was defined as the main key for taking decisions and ATOMAC guidelines gained support. However, facing a blunt trauma in children is always a challenge. Hemodynamic stability is not easy to define and heart rate and blood pulse are poor markers for stability. This complicates the management of blunt trauma in children. As consequence, it is important that these children are treated in pediatric centers. We report a case of a 15-year-old male who suffered from a blunt abdominal trauma with splenic and renal injuries treated by adult surgeons when playing football. We review the current recommendations for blunt abdominal trauma in children and the differences with adult management.
\end{abstract}

Keywords: Pediatric trauma; Splenic injury; Renal injury; Abdominal trauma; Blunt trauma

\section{Introduction}

Intraabdominal injuries occur as a result of blunt abdominal trauma (BAT) or penetrating trauma, being BAT the most common cause $(85 \%)$ and penetrating trauma accounting for only a

Manuscript submitted August 29, 2017, accepted September 13, 2017

a General and Digestive Surgery Department, Principe de Asturias Hospital, Madrid, Spain

${ }^{b}$ Corresponding Author: Ana Maria Minaya-Bravo, General and Digestive Surgery Department, Principe de Asturias Hospital, Madrid, Spain.

Email: ana-minaya@hotmail.com

doi: https://doi.org/10.14740/jmc2901w minority $(15 \%)$. Abdominal trauma is the third cause of death in children over 1 year of age $[1,2]$. Intraabdominal injuries are present in $5-10 \%$ of all BATs $[2,3]$. Approximately 50$75 \%$ of BATs are caused by motor vehicle collisions. Other causes are sport injuries, falls and child abuse. Sport injuries usually cause isolated organ injury $[1,2]$.

There are two main mechanisms: direct blow to abdomen and high-energy mechanisms that produce multi-system trauma. The latter is associated with higher mortality [3, 4]. Solid organ injury (SOI) is referred to liver, spleen or renal. The most frequent organs injured are spleen, liver and kidney; being spleen the most frequent cause of intraabdominal bleeding. Although blunt renal injuries are much less common than splenic or liver lesions, children are more susceptible to renal injuries than adults because of anatomical aspects [5].

The American Association for the Surgery of Trauma (AAST) grading is the most used for classification of splenic, liver and renal injuries (Fig. 1) [6, 7]. The management of SOI has evolved since 1970 when a group of pediatric surgeons in Toronto advocated for non-operative management (NOM) of splenic injuries. This new tendency has been accompanied by the advance of diagnostic imaging techniques that has allowed to achieve a more precise evaluation of the lesion and to avoid delay in treatment. However, management of blunt trauma in children has been motive of debate in the past years. Currently, hemodynamic status is considered the most relevant factor when taking decisions [8, 9].

NOM of solid organ injuries, mainly liver and spleen, reaches a successful rate over $90 \%$ and for blunt renal injuries around $84-89 \%[10,11]$. We report a case of a 15 -year-old male that suffered from blunt trauma when playing football with both kidney and splenic injuries. We discuss and review the challenge of management of blunt trauma in pediatric patients, as well as, the current guidelines.

\section{Case Report}

A 15-year-old male with no relevant medical history was admitted to the emergency department due to abdominal pain of acute onset after BAT.

The patient reported to be playing football and suffer a direct blow on his upper left side of abdomen.

On admission, the patient was alert and responsive, the blood pressure was $80 / 50 \mathrm{~mm} \mathrm{Hg}$ and pulse was 110 beats per minute. Upon physical examination, he did not present any ex- 

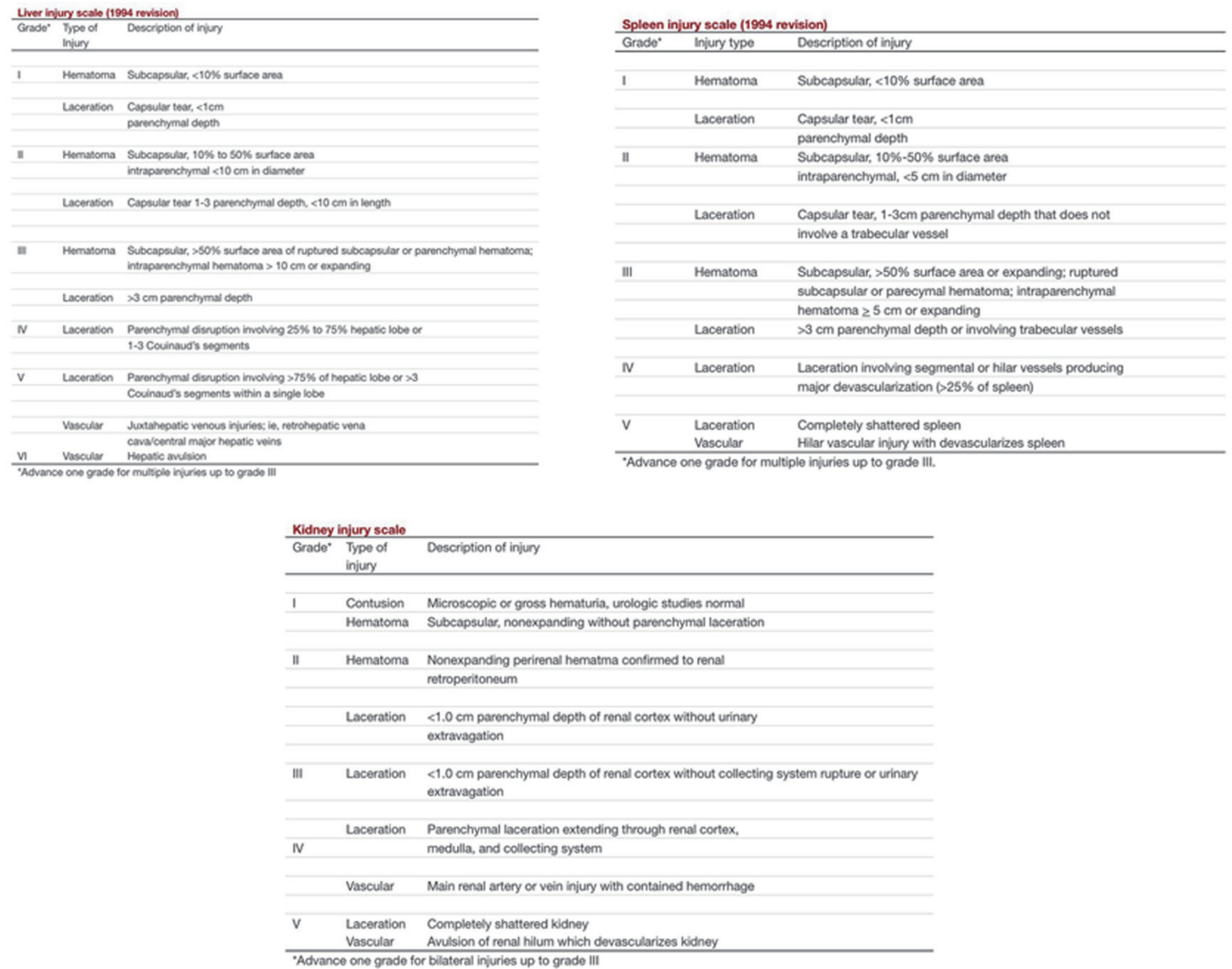

Figure 1. AAST grading of liver, spleen and renal injuries.

ternal lesion and was found to have diffuse abdominal pain with tenderness on the left hypochondriac region and left flank.

The laboratory test results revealed hemoglobin $14.3 \mathrm{mg} /$ $\mathrm{dL}$ and white blood cell count $13,000 / \mathrm{mm}^{3}$. Focused assessment with sonography in trauma (FAST) was positive for free fluid in abdomen. After initial resuscitation with crystalloid fluids, blood pressure increased to $100 / 80 \mathrm{~mm} \mathrm{Hg}$ and computerized tomography (CT) was performed.

CT demonstrated intraabdominal fluid with a grade III splenic injury with hemoperitoneum and, in addition, a grade III renal injury (Fig. 2). The patient underwent urgent surgery due to tendency to hypotension and tachycardia. A middle laparotomy was chosen as surgical approach and a splenic laceration of more than $3 \mathrm{~cm}$ near splenic vessels was found, with hemoperitoneum of $2 \mathrm{~L}$ and retroperitoneal hematoma due to renal injury.

We decided to perform a conservative surgery of spleen, and applied human-derived thrombin product. None surgical action was done on the retroperitoneal hematoma. The patient was transfused two packed red blood cells during surgery and admitted to intensive care unit (ICU) where he stayed for 2 days. He remained stable without active bleeding signs. We advised bedrest for these 2 days. Then, he was transferred to ward.
On the seventh postoperative day, a CT follow-up showed healing of splenic and renal injuries with no free fluid. Hemoglobin remained stable and he was discharged (Fig. 3). We advised him walking but restriction of any kind of sport for 1 month. Two months after trauma a US was carried out and demonstrated complete resolution of the injuries (Fig. 4).

The patient did present neither hypertension nor hematuria during the process. Nowadays, he is doing well.

\section{Discussion}

Management of SOIs has been object of discussion for decades, and has undergone a radical shift in the last years. Currently, NOM is the standard care [12-14]. In 1971, in Toronto, Douglas et al reported a series of 32 cases of splenic injuries, of which 26 were successfully treated with conservative management and only six required surgery [8]. Later, in 1977, Aronson et al published a short series of children with splenic trauma treated without surgery [9]. As a result, controversy about management of splenic trauma arose: non-operative versus operative management.

In 2000, APSA supported NOM and established consensus about liver and splenic blunt trauma, and this included that 

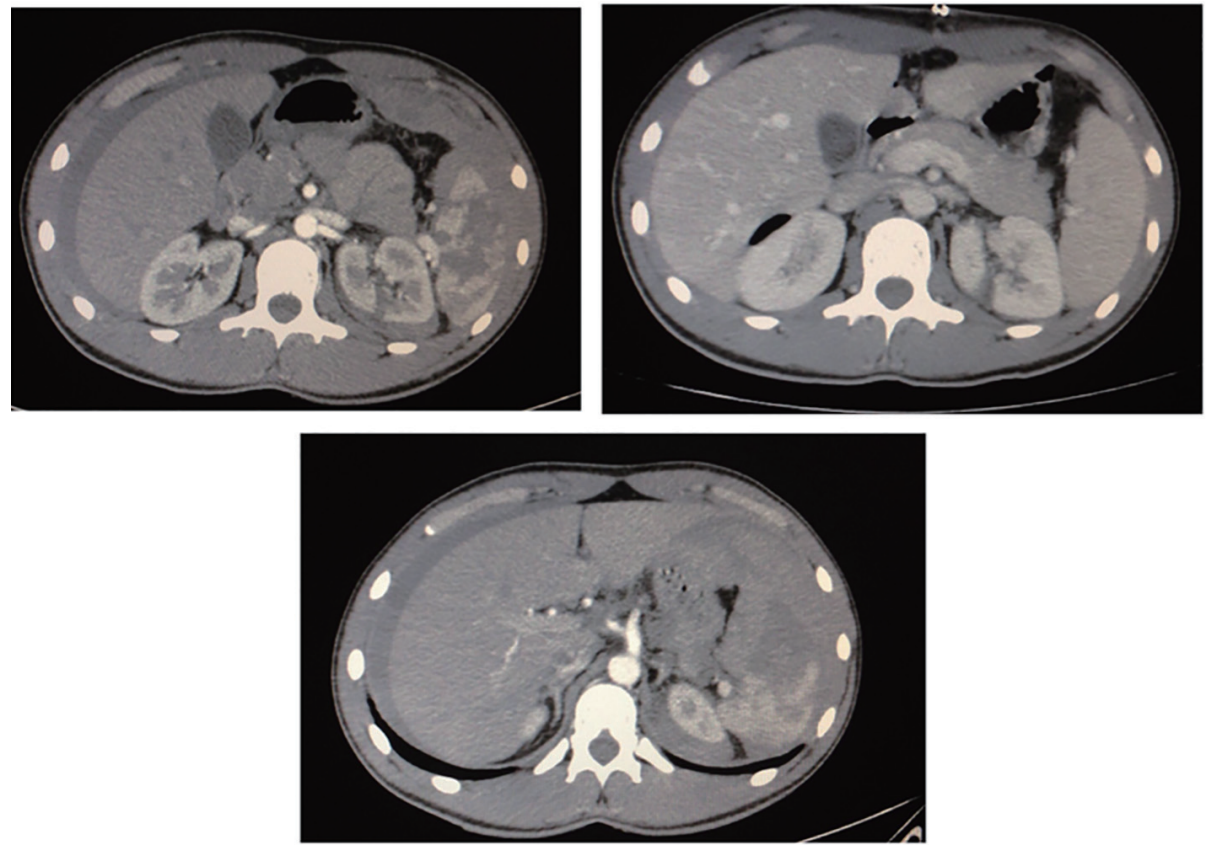

Figure 2. CT showed grade III splenic injury with splenic laceration more than $3 \mathrm{~cm}$ and hematoma more than $50 \%$ : irregular hypodense area with indistinct margins, which is not enhanced after contrast medium administration. Kidney presented irregular non-enhancing renal parenchyma defect more than $1 \mathrm{~cm}$ in depth. Collecting system was not affected. That was considered grade III renal injury. Free intraabdominal blood was evidenced.

all IV grade injuries required ICU admission, independently of hemodynamic status. Moreover, hospitalization time depended on injury grade, so they recommended a hospital stay of "grade of injury plus one" in days (in other words, grade III, hospital stay of 4 days). Return to normal activity was also based on grade injury: "two weeks plus grade of lesion" (for example, grade II, 4 weeks of activity restriction). APSA guidelines were based on image findings and grading according to APSA. This was also extended to renal injuries $[6,7,15]$.

However, the controversy continued, and APSA guidelines were questioned. The fact that APSA decisions depended, mainly, on images created an excessive hospitalization and consequently, high costs, long periods of bedrest and unneces-



Figure 3. CT on the seventh postoperative day demonstrated healing of splenic laceration with hyperdense and isodense areas in spleen, and healing of renal laceration. No free intraperitoneal blood was shown. sary imaging follow-up.

In 2008, McVay et al cast doubt on the need of ICU admission based only on CT findings, and pointed that decision taking must be guided by hemodynamic status [16]. As a result of this new perspective of management, a consortium of American College of Surgeons performed a systematic review of the literature to reach conclusions of management. In consequence, in 2012, experts on pediatric trauma developed Arizona-Texas-Oklahoma-Memphis-Arkansas Consortium (ATOMAC) guidelines. ATOMAC guidelines provided new algorithms and were adopted in many pediatric centers (Fig. 5 ). They emphasized the role of hemodynamic status as main factor for decision taking [17]. In 2015, Notrica et al reported a review of more than 300 papers and concluded that only patients with signs of recent or ongoing bleeding require ICU admission. Again, physiological parameters are crucial. Moreover, grade 5 injuries may benefit from ICU admission $[13,17]$.

It has been reported that shock index adjusted for children (SIPA) and hematocrit over $30 \%$ predict what children that can be safely treated on ward and, in consequence, reduce costs. Similarly, the absence of these criteria allows selecting children that will require ICU admission [12].

As a result, NOM has been established as the gold standard treatment for solid organ injury. Experience and accurate diagnosis are crucial to implement conservative treatment [13, $14,17,18]$.

Double contrast CT is a useful tool to identify arterial extravasation of contrast and hemoperitoneum. However, it can only be performed safely on stable patients. Unstable patients should undergo FAST. In case of positive FAST and hemodynamic stability, a CT should be taken, since FAST does not 

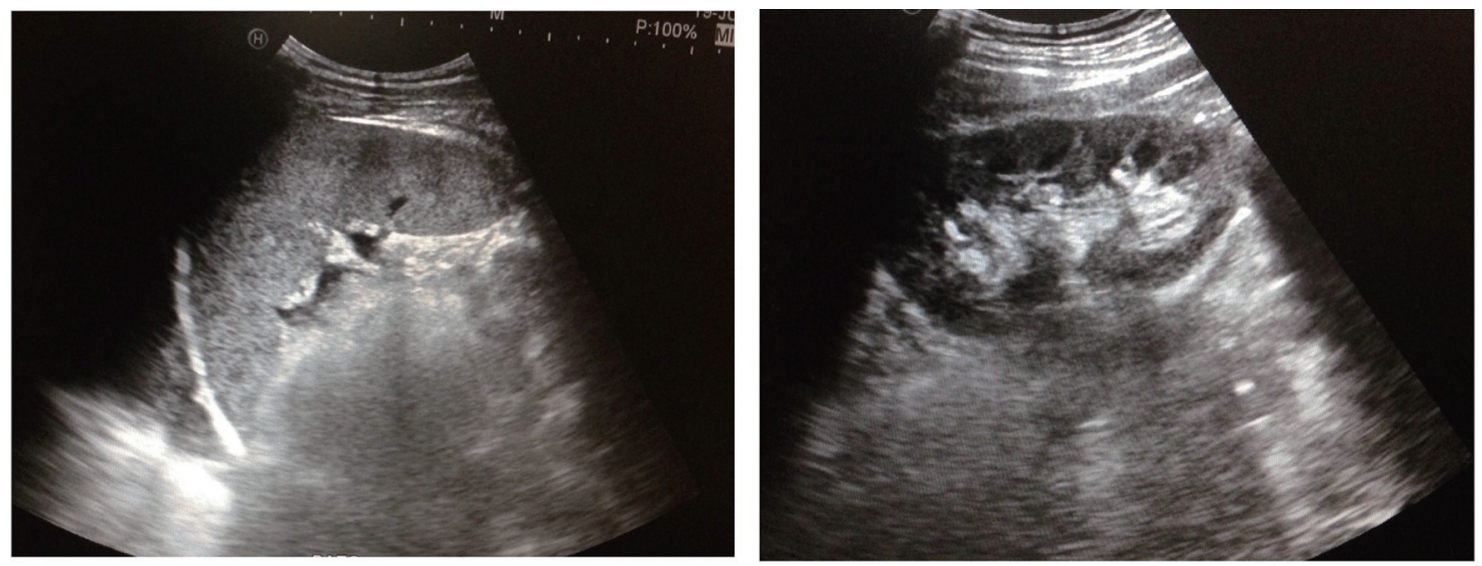

Figure 4. US showed complete resolution: on the left spleen and on the right kidney.

replace CT $[1,19,20]$.

The concern of radiation and cancer in children has made other alternatives to emerge: for instance, contrast enhanced ultrasound (CEUS) that uses perflutren gas (Optison) as contrast [21]. CEUS has proven to have a sensitivity of $96 \%$ and specificity of $99 \%$ in children and adults for identifying trauma injuries [22]. Moreover, combination of SIPA and mechanism of lesion could select patients that will require $\mathrm{CT}$ and avoid unnecessary CTs [23].
The initial management of blunt SOI is based on critical principles of resuscitation: airway, breathing and circulation. Resuscitation must be based on crystalloid fluids and transfusion when required. The use of crystalloid fluids must be limited, as in adult management. Transfusion over $40 \mathrm{~mL} / \mathrm{kg}$ is associated with high mortality. Transfusion threshold is $7.0 \mathrm{~g} /$ $\mathrm{dL}$ for children under NOM. There is lack of evidence about transfusions improve the success of NOM [17]. Management of patients that show signs of ongoing bleeding differs from those

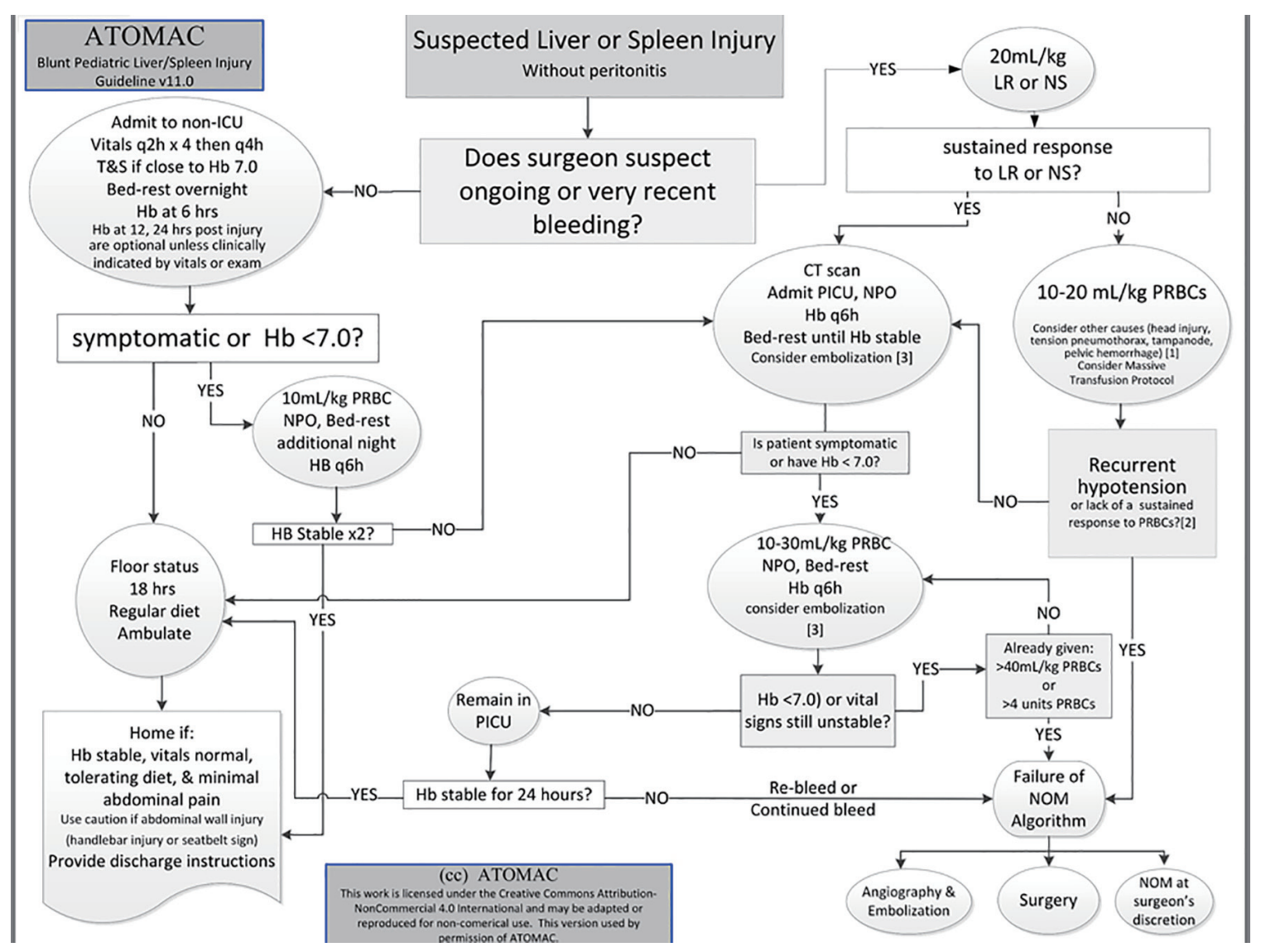

Figure 5. ATOMAC algorithm. 
without bleeding signs and is based on hemodynamic state. Once the patient responds to resuscitation and stability has been achieved, CT with intravenous contrast must be performed.

In those children that do not respond to fluid therapy the need of transfusion must be evaluated individually; this situation always supposes a challenge for the surgeon. The decision will depend on several factors: injuries, type of center, radiology department and experience. Under these circumstances, the clinician will have to determine the best option: surgery, angiography and even NOM [18]. However, it is difficult to define stability in children. Half of the patients who are unstable are not bleeding; on contrary, many children who are bleeding are not unstable. Furthermore, neither blood pressure nor heart rate is good markers of hemorrhagic shock [14, 24]. Stable hemoglobin has been defined as not dropping more than $0.5 \mathrm{~g} / \mathrm{dL}$ in $24 \mathrm{~h}$ [17]. Some pediatric shock indexes have been reported to evaluate shock. Also, it must be taken into account that head trauma is a common cause of hypotension $[17,25]$.

In the last decade, operative management of SOI has plummeted. Currently, it is estimated that in pediatric hospitals the rate of laparotomies due to failure of NOM of SOI is $3.2 \%$. The most common cause of failure of NOM is hemorrhage and persistent hypovolemic shock [26]. Embolization is an option for SOI, especially when contrast extravasation is demonstrated [17]. The Committee on Trauma of the American College of Surgeons supports the use of embolization in pediatric trauma centers and many authors have already reported successful results in highly qualified centers [27, 28].

Current bedrest recommendations differ from those from APSA guidelines. For stable patients, 1 day or even less of bedrest is enough once there is documentation of stable hemoglobin. It has been demonstrated that rebleeding is exceptional, with an incidence of $0.3 \%$. Furthermore, there is no evidence about benefit of bedrest and rebleeding. So, many patients with SOI could be discharged before 24 hours, as long as family are instructed on signs and symptoms of bleeding [13, 17]. Moreover, follow-up imaging after discharge is not usually recommended, as it occasionally shows no clinical important findings that may conduct to more unnecessary reimaging [17, 13, 29].

Renal blunt injuries are rare lesions that account for $1 \%$ of blunt trauma. NOM of liver and spleen injuries has been applied to blunt renal trauma. Even injury of collecting system with urine extravasation can be treated with non-aggressive techniques, for example percutaneous nephrostomy or stents $[10,30]$.

There is consensus of NOM for low grade renal injuries; however, high grade injuries are surrounded by controversy; although, there is a clear tendency to NOM $[11,30]$.

Graziano et al on his paper demonstrated that decision taking for renal blunt injuries was also based on hemodynamic status and bedrest was not required [5]. Recently, LeeVan et al reported a systematic retrospective review of 32 papers, in which they found minimal evidence to support ICU admission for high grade renal lesions, similarly to papers for SOI. In the same way, the evidence of need for urinary catheter, prophylactic antibiotics and bedrest is insufficient to be recommended. US seems to be a proper method for evaluation of evolution. Again, hemodynamic factor plays the most important role when deciding surgery, regardless type of lesion.
Similarly, discharge should be based on hemodynamic status with no routine imaging techniques recommended. Occasionally, blood pressure monitoring could be planned. About complications, in case of urinoma, percutaneous drainage is a good option [31].

We highlight that our center (level 2) lacks of Pediatric Department, so our patient was treated by adult general surgeons. We faced a patient with tendency to hemodynamic instability after resuscitation and hemoperitoneum on CT with a III grade splenic injury and III grade renal injury. On admission, he presented a hemoglobin level of $14.3 \mathrm{~g} / \mathrm{dL}$. Instability and hemoperitoneum, mainly, made us to decide surgery as best option. The absence of radiologist on clock excluded the possibility of embolization. We are aware that the management of this patient may have been different in a pediatric center. Carrie et al estimated that adult surgeons are almost 15 times more likely to go surgery in stable patient when compared to pediatric surgeons, however, mortality does not differ in both cases [28].

The tendency to hypotension and hemoperitoneum put us in a challenging situation and eventually, decided to go to surgery, not for renal lesion but splenic injury. However, once in operating room, we found hemoperitoneum and grade III splenic injury, on that moment we decided to be more conservative and avoided splenectomy, we only performed hemostasis with human-derived thrombin product. Moreover, he was transfused two units of packed red blood. The easiest decision had been to remove the spleen; however, we wanted to avoid consequences as post splenectomy infection. We overestimated blood pressure as marker of instability, probably because we are not used to deal with children. In addition, we probably rushed, unnecessarily, for transfusion. According to Partrick et al, hypotension in injured children is not often associated with bleeding insult, especially in children under 5 years old, and head trauma is a frequent cause of hypotension [24]. The patient underwent follow-up imaging and a CT was performed before discharge that showed an organized hematoma and healing. Two months later, a US was performed.

According to current guidelines follow-up imaging is unnecessary [17]. However, we were too much cautious and performed two imaging techniques for follow-up, probable because we are not used to deal with children and overestimated the situation.

It has been proved that non-pediatric hospitals have a higher rate of operative treatment than pediatric units, and splenectomy rate is considered a marker of care quality $[10,32]$. We conclude that when possible injured children must be managed in pediatric centers. If not possible, then adult surgeons must have in mind that physiological parameters in children differ from adults and management is always a challenge. To define stability in children is difficult and no rush decisions must be taken. NOM is always the standard care when circumstances are favorable.

\section{Conclusion}

Management of SOI has been in a continuous review and controversy in the last decades. Currently, NOM is the stand- 
ard care for stable injuries and it is hemodynamic status factor that will conduct decision taking. However, children are a challenge: to define hemodynamic stability is not easy and although, several indicators have been proposed until now, there is not a single marker that indicates instability. Hypotension and heart rate are not reliable markers to define stability. Other factor added to this challenge, is the concern of radiation and CT in children, especially in patients under 10 years old that present the highest risk.

The successful of NOM is higher in centers with Pediatric Department, it has been proved that surgeons that usually do not deal with children are more inclined to surgical treatment because of the lack of experience in determine and evaluate hemodynamic status in children. ATOMAC guideline has been adopted in many pediatric centers and, currently, offers good results

\section{Grant Support}

None.

\section{Conflict of Interest}

None.

\section{References}

1. Djordjevic I, Slavkovic A, Marjanovic Z, Zivanovic D. Blunt trauma in paediatric patients - experience from a small centre. West Indian Med J. 2015;64(2):126-130.

2. Drexel S, Azarow K, Jafri MA. abdominal trauma evaluation for the pediatric surgeon. Surg Clin North Am. 2017;97(1):59-74.

3. Gaines BA. Intra-abdominal solid organ injury in children: diagnosis and treatment. J Trauma. 2009;67(2 Suppl):S135-139.

4. Pariset JM, Feldman KW, Paris C. The pace of signs and symptoms of blunt abdominal trauma to children. Clin Pediatr (Phila). 2010;49(1):24-28.

5. Graziano KD, Juang D, Notrica D, Grandsoult VL, Acosta J, Sharp SW, Murphy JP, et al. Prospective observational study with an abbreviated protocol in the management of blunt renal injury in children. J Pediatr Surg. 2014;49(1):198-200; discussion 200-191.

6. Buckley JC, McAninch JW. Revision of current American Association for the Surgery of Trauma Renal Injury grading system. J Trauma. 2011;70(1):35-37.

7. Moore EE, Shackford SR, Pachter HL, McAninch JW, Browner BD, Champion HR, Flint LM, et al. Organ injury scaling: spleen, liver, and kidney. J Trauma. 1989;29(12):1664-1666.

8. Douglas GJ, Simpson JS. The conservative management of splenic trauma. J Pediatr Surg. 1971;6(5):565-570.

9. Aronson DZ, Scherz AW, Einhorn AH, Becker JM, Schneider KM. Nonoperative management of splenic trauma in children: a report of six consecutive cases. Pediatrics. 1977;60(4):482-485.

10. Nance ML, Lutz N, Carr MC, Glendemab JN, Myers RL. Blunt renal injuries in children can be managed non-operatively: outcome in a consecutive series of patients. $\mathrm{J}$ Trauma. 2004;57:474e8.

11. Margenthaler JA, Weber TR, Keller MS. Blunt renal trauma in children: experience with conservative management at a pediatric trauma center. J Trauma. 2002;52(5):928932.

12. Arbuthnot M, Armstrong LB, Mooney DP. Can we safely decrease intensive care unit admissions for children with high grade isolated solid organ injuries? Using the shock index, pediatric age-adjusted and hematocrit to modify APSA admission guidelines. J Pediatr Surg. 2017;52(6):989-992.

13. Notrica DM. Pediatric blunt solid organ injury: beyond the APSA guidelines. Curr Surg Rep. 2015;3:7.

14. Notrica DM. Pediatric blunt abdominal trauma: current management. Curr Opin Crit Care. 2015;21(6):531-537.

15. Stylianos S. Evidence-based guidelines for resource utilization in children with isolated spleen or liver injury. The APSA Trauma Committee. J Pediatr Surg. 2000;35(2):164-167; discussion 167-169.

16. McVay MR, Kokoska ER, Jackson RJ, Smith SD. Throwing out the "grade" book: management of isolated spleen and liver injury based on hemodynamic status. J Pediatr Surg. 2008;43(6):1072-1076.

17. Notrica DM, Eubanks JW, 3rd, Tuggle DW, Maxson RT, Letton RW, Garcia NM, Alder AC, et al. Nonoperative management of blunt liver and spleen injury in children: Evaluation of the ATOMAC guideline using GRADE. J Trauma Acute Care Surg. 2015;79(4):683-693.

18. Notrica DM, Linnaus ME. Nonoperative management of blunt solid organ injury in pediatric surgery. Surg Clin North Am. 2017;97(1):1-20.

19. Fang JF, Wong YC, Lin BC, Hsu YP, Chen MF. Usefulness of multidetector computed tomography for the initial assessment of blunt abdominal trauma patients. World $\mathrm{J}$ Surg. 2006;30(2):176-182.

20. Armstrong LB, Mooney DP, Paltiel H, Barnewolt C, Dionigi B, Arbuthnot M, Onwubiko C, et al. Contrast enhanced ultrasound for the evaluation of blunt pediatric abdominal trauma. J Pediatr Surg. 2017.

21. Miglioretti DL, Johnson E, Williams A, Greenlee RT, Weinmann S, Solberg LI, Feigelson HS, et al. The use of computed tomography in pediatrics and the associated radiation exposure and estimated cancer risk. JAMA Pediatr. 2013;167(8):700-707.

22. Sessa B, Trinci M, Ianniello S, Menichini G, Galluzzo M, Miele V. Blunt abdominal trauma: role of contrastenhanced ultrasound (CEUS) in the detection and staging of abdominal traumatic lesions compared to US and CEMDCT. Radiol Med. 2015;120(2):180-189.

23. Acker SN, Stewart CL, Roosevelt GE, Partrick DA, Moore EE, Bensard DD. When is it safe to forgo abdominal CT in blunt-injured children? Surgery. 2015;158(2):408-412.

24. Partrick DA, Bensard DD, Janik JS, Karrer FM. Is hypotension a reliable indicator of blood loss from traumatic 
injury in children? Am J Surg. 2002;184(6):555-559; discussion 559-560.

25. Acker SN, Ross JT, Partrick DA, Tong S, Bensard DD. Pediatric specific shock index accurately identifies severely injured children. J Pediatr Surg. 2015;50(2):331334.

26. Wisner DH, Kuppermann N, Cooper A, Menaker J, Ehrlich P, Kooistra J, Mahajan P, et al. Management of children with solid organ injuries after blunt torso trauma. J Trauma Acute Care Surg. 2015;79(2):206-214;quiz 332.

27. Sweed Y, Singer-Jordan J, Papura S, Loberant N, Yulevich A. Angiographic embolization in pediatric abdominal trauma. Isr Med Assoc J. 2016;18(11):665-668.

28. Sims CA, Wiebe DJ, Nance ML. Blunt solid organ injury: do adult and pediatric surgeons treat children differently? J Trauma. 2008;65(3):698-703.
29. Safavi A, Beaudry P, Jamieson D, Murphy JJ. Traumatic pseudoaneurysms of the liver and spleen in children: is routine screening warranted? J Pediatr Surg. 2011;46(5):938-941.

30. Burns J, Brown M, Assi ZI, Ferguson EJ. Five-year retrospective review of blunt renal injuries at a level i trauma center. Am Surg. 2017;83(2):148-156.

31. LeeVan E, Zmora O, Cazzulino F, Burke RV, Zagory J, Upperman JS. Management of pediatric blunt renal trauma: A systematic review. J Trauma Acute Care Surg. 2016;80(3):519-528.

32. Hsiao M, Sathya C, de Mestral C, Langer JC, Gomez D, Nathens AB. Population-based analysis of blunt splenic injury management in children: operative rate is an informative quality of care indicator. Injury. 2014;45(5):859-863. 